\title{
XXIII.
}

\section{SUL PRINCIPIO DI DIRICHLET}

\author{
"Circolo Mat. di Palermo », vol. XI, I897, pp. 83-86 (*).
}

I. In quasi tutti i corsi sulla teoria del potenziale newtoniano o logaritmico si suole esporre la dimostrazione di NEUMANN sul principio di DIRICHLET $^{(x)}$.

Ma nella trattazione del detto metodo si incontra ordinariamente una difficoltà, la quale, senza infirmare il metodo stesso, può dar luogo a qualche oscurità.

In un corso di lezioni del I $^{8} 88^{(2)}$ tentai superarla, ed alcuni miei colleghi avendo sperimentato favorevolmente la osservazione che feci in proposito desiderarono che venisse pubblicata, il che mi permetto di far qui brevemente.

Noterò ancora che ci si può valere della stessa osservazione nell'esporre il metodo di RoBIN per la determinazione della densità d'uno strato di livello ${ }^{(3)}$ e così in alcune altre questioni analoghe.

2. Non starò a spiegare in che cosa consista la dimostrazione di NEUMANN e mi varrò senz'altro della denominazione di funzione aggiunta ormai generalmente adottata.

Col metodo di NEUMANN si giunge a provare che se $U$ è una funzione finita e continua data sul contorno convesso e $\mathrm{P}$ è la sua aggiunta, si ha

$$
\frac{\text { oscillazione } \mathrm{P}}{\text { oscillazione } \mathrm{U}}<\lambda<\mathrm{I} \text {, }
$$

in cui $\lambda$ è un numero indipendente dalla funzione $U$, ma che dipende solo dalla forma del contorno.

Tale dimostrazione è pienamente rigorosa quando è possibile dividère il contorno in un numero finito di pezzi in alcuni dei quali $U$ è superiore al suo valore medio ${ }^{(4)}$, mentre negli altri è inferiore o eguale al valore medio stesso.

$\left(^{*}\right)$ Presentata nell'adunanza del 28 febbraio 1897.

(1) Über die Methode des aritmetischen Mittels. "Abhand. d. math.-phys. Classe d. Königl. Sächs. Gesell. d. Wissenschaften ", Bd. XIII, p. 705, I887, Bd. XIV, p. 563, I888.

(2) In quell'anno mi limitai al potenziale logaritmico. Il dott. Bigiavi in un corso successivo applicò lo stesso procedimento al potenziale newtoniano.

(3) "Comptes Rendus des séances de l'Académie des Sciences", t. CIV, p. 1834, 1887.

(4) Per valore medio intendo qui la media fra il massimo ed il minimo valore della funzione. 
Ma nel caso del potenziale logaritmico in cui il contorno è una linea si vede facilmente che questo fatto potrà non presentarsi quando la funzione $\mathrm{U}$ fa un numero infinito di oscillazioni pur essendo continua, e nel caso del potenziale newtoniano si comprende facilmente che la detta divisione in due parti del contorno darà luogo a difficoltà ancora maggiori.

Per togliere nelle dimostrazioni ogni dubbio, che perciò potrebbe presentarsi, basterà provare che ogni funzione finita e continua $U$ può mettersi sotto la forma di una serie convergente in egual grado

$$
\mathrm{U}=\sum_{\mathrm{I}}^{\infty} \mathrm{U}_{i}
$$

in cui per ciascuna $\sum_{I}^{n} U_{i}$ può eseguirsi la divisione del contorno in un numero finito di parti, in alcune delle quali $\sum_{I}^{n} U_{i}$ sia maggiore del suo valor medio, mentre nelle rimanenti è minore o eguale al detto valore; giacché, una volta dimostrato questo, avremo che la serie delle funzioni aggiunte $\mathrm{P}=\sum_{I}^{\infty} \mathrm{P}$ sarà pure uniformemente convergente, e

$$
\frac{\text { oscillazione }}{\text { oscillazione } \sum_{\mathrm{I}}^{n} \mathrm{P}_{i}}<\lambda
$$

quindi il rapporto

$$
\frac{\text { oscillazione } \mathrm{P}}{\text { oscillazione } \mathrm{U}}=\frac{\text { oscillazione } \sum_{\mathrm{I}}^{n} \mathrm{P}_{i}}{\text { oscillazione } \sum_{\mathrm{I}}^{n} \mathrm{U}_{i}}
$$

non potrà superare $\lambda$.

3. Ora il detto teorema, nel caso in cui il contorno in cui è definito $U$ è lineare, si dimostra cosi.

$\mathrm{La} \mathrm{U}$ può ritenersi come una funzione di una variabile $x$ definita in un intervallo $(a b)$; onde, considerando $x, y$ come un sistema di coordinate cartesiane, $y=\mathrm{U}(x)$ rappresenterà una linea $\mathrm{L}$ nel piano $x, y$.

Dividiamo $(a b)$ in $n_{i}$ parti in modo che entro ciascuna di esse $U$ faccia una oscillazione minore di $\sigma / i$. Prendiamo i punti di $\mathrm{L}$ che hanno per proiezione sull'asse $x$ 'i punti di divisione, consideriamo la spezzata che ha per vertici i detti punti di L, e chiamiamo $y=f_{i}(x)$ la sua equazione. Allora la serie (I) sarà subito costruita prendendo

$$
\mathrm{U}_{i}=f_{i}-f_{i-1} \quad, \quad f_{\mathrm{o}}=\mathrm{o} .
$$

Nel caso in cui il contorno sia una superficie convessa proiettiamola da un punto interno sopra una superficie sferica avente il centro in quel punto. 
Potremo evidentemente considerare $U$ come una funzione dei punti della superficie sferica e, determinando questi punti mediante la latitudine $\theta$ e la longitudine $\varphi$, potremo considerare $U$ come una funzione di $\theta$ e $\varphi$. Supponendo poi che $\theta, \varphi, z$ siano un sistema di coordinate cartesiane, avremo che $z=\mathrm{U}(\theta, \varphi)$ rappresenterà una superficie che si proietterà sul piano $\theta$, $\varphi$ secondo un rettangolo di lati rispettivamente eguali a $\pi$ e a $2 \pi$.

Si divida questo rettangolo colle parallele agli assi in $n_{i}$ rettangoli in modo che corrispondentemente a ciascuno di essi la oscillazione della funzione $z=\mathrm{U}(\theta, \varphi)$ sia minore di $\sigma / i^{(5)}$ e si inscriva entro la superficie una superficie poliedrica a facce triangolari i cui vertici si proiettino nei punti di divisione. Se $z=f_{i}(\theta, \varphi)$ è l'equazione di questa superficie poliedrica, si otterrà la serie $(\mathrm{I})$ prendendo $\mathrm{U}_{i}=f_{i}-f_{i-1}, f_{\mathrm{o}}=0$.

4. La dimostrazione fatta nel paragrafo precedente nel caso del contorno lineare consiste nel dedurre dal principio della continuità uniforme la proprietà che ha qualsiasi funzione finita e continua di potersi considerare come limite di una funzione finita e continua avente un numero limitato di massimi e di minimi, la quale tende uniformemente alla prima.

Questa proprietà giova in vari casi, oltre che nella dimostrazione del principio di DiRICHLET. Cosi, per esempio, tenendo conto che una funzione finita e continua che ha un numero limitato di massimi e di minimi è sviluppabile in serie di FOURIER uniformemente convergente, potremo concludere senz'altro che ogni funzione finita e continua ammette una rappresentazione mediante un polinomio finito di FOURIER con una approssimazione data qualsiasi.

Questo bel teorema che il PICARD ${ }^{(6)}$ deduce dall'integrale di PoIsson e dal quale egli fa dipendere una celebre proposizione di WEIERSTRASS risulta così provato in via immediata e diretta.

Torino, 15 febbraio 1897 .

(5) Ciò potrà farsi giacchè le funzioni continue di più variabili sono, al pari di quelle ad una variabile, uniformemente continue.

(6) Traité d'Analyse, Tome I, page 257. 